\title{
A Interface entre as Políticas Públicas de Saúde Mental e Promoção da Saúde'
}

The Interface Between the Mental Health and Health

\section{Promotion Public Policies}

\author{
Juliana Reale Caçapava \\ Enfermeira, Mestre em Enfermagem Psiquiátrica. \\ Endereço: Av. Dr. Enéas Carvalho de Aguiar, 419, Cerqueira César, \\ CEP 05403-000, São Paulo, SP, Brasil. \\ E-mail: jurealeœuol.com.br \\ Luciana de Almeida Colvero \\ Professora Doutora da Escola de Enfermagem da Universidade de \\ São Paulo (EEUSP). \\ Endereço: Av. Dr. Enéas Carvalho de Aguiar, 419, Cerqueira César, \\ CEP 05403-000, São Paulo, SP, Brasil. \\ E-mail: lucixळusp.br \\ Isabel Maria Teixeira Bicudo Pereira \\ Professora Doutora da Faculdade de Saúde Pública da Universi- \\ dade de São Paulo. \\ Endereço: Av. Dr. Arnaldo 715, Cerqueira César, CEP 01246-904, \\ São Paulo, SP, Brasil. \\ E-mail: claiannyळusp.br \\ I A pesquisa foi financiada pela Fundação de Amparo à Pesquisa \\ do Estado de São Paulo (FAPESP).
}

\section{Resumo}

Este estudo tem por objetivo analisar as estratégias de produção de cuidado em saúde mental de um Centro de Convivência e Cooperativa (CECCO) - serviço da rede de atenção à saúde mental do município de São Paulo - na perspectiva de seu alinhamento às políticas de saúde mental e promoção da saúde, verificando as potencialidades da interação entre elas, no cotidiano das práticas de saúde. A análise baseou-se no material empírico obtido por meio de entrevista semiestruturada junto ao gerente do serviço estudado, assim como nos achados da literatura e nos documentos oficiais do Ministério da Saúde. Verificou-se que, por meio de ações intersetoriais, que visam ao fortalecimento da participação social, do empoderamento de indivíduos e comunidade e da equidade, o CECCO articula pressupostos da saúde mental e da promoção da saúde, produzindo autonomia e cidadania e tornando-se um lugar que constrói sentido de vida às pessoas.

Palavras-chave: Saúde mental; Promoção da saúde; Serviços comunitários de saúde mental. 


\section{Abstract}

This study aimed at analyzing the mental health care production strategies of a 'Centro de Convivência e Cooperativa' (CECCO) - a service of the mental health care network of the city of São Paulo - in the perspective of their alignment with the mental health and health promotion public policies, verifying the potentialities of the interaction between them in the health practices' daily routine. The analysis was based on the empirical material obtained by a qualitative research, through a semi-structured interview with the health service's manager, as well as on the literature's findings and on the official documents of the Ministry of Health. It was observed that, through intersectorial actions aiming at the strengthening of social participation, of the empowerment of individuals and community and of equity, CECCO articulates the presuppositions of mental health and of health promotion, producing autonomy and citizenship and becoming a place that constructs life's meaning to people.

Keywords: Mental Health; Health Promotion; Community-Based Mental Health Services.

\section{Introdução}

Este trabalho busca desenvolver uma discussão entre saúde mental e promoção da saúde, sendo oriundo de pesquisa realizada em um Distrito de Saúde do município de São Paulo, em 2006, na qual buscávamos caracterizar o atendimento de saúde mental de certo território (Caçapava e Colvero, 2008).

$\mathrm{Na}$ ocasião, observamos as fragilidades das Unidades Básicas de Saúde (UBS) na articulação com os equipamentos sociais do território e com a comunidade, ou seja, na construção e na operacionalização de uma rede de atenção de base comunitária em saúde mental, que deve ser constituída, além dos serviços de saúde, de equipamentos jurídicos, de lazer, cultura, educação, trabalho etc.

Em contrapartida, verificamos que um dos serviços que compõem essa rede de atenção em saúde mental no território estudado, o Centro de Convivência e Cooperativa (CECCO), de forma coerente às ações e iniciativas da política de saúde mental do SUS, desenvolve estratégias de cuidado no sentido de fortalecer os laços de inclusão social dos sujeitos portadores de sofrimento psíquico, buscando parcerias para desenvolver ações de saúde pautadas pela integralidade - pela vertente do trabalho, da cultura, do lazer, da convivência - e, portanto, operando através da intersetorialidade, diretriz essencial da promoção da saúde.

Assim, através das ações intersetoriais, o alinhamento do CECCO à promoção da saúde fomentou-nos a reflexão sobre as potencialidades da interação entre saúde mental e promoção da saúde, mediante as políticas públicas que se reportam a elas no país, e com as quais buscamos dialogar neste artigo, direcionando nosso foco a três significativos documentos publicados pelo Ministério da Saúde: $3^{a}$. Conferência Nacional de Saúde Mental: cuidar sim, excluir, não; Política Nacional de Promoção da Saúde e Saúde Mental no SUS: acesso ao tratamento e mudança do modelo de atenção; Relatório de gestão 2003-2006 (Brasil, 2002, 2006, 2007).

A partir de seus princípios de integralidade, universalidade, equidade, descentralização e participação da comunidade, o Sistema Único de Saúde (SUS), reconheceu muitos direitos de cidadania, afirmando que não é possível produzir saúde numa perspectiva restrita, sem considerar todos os processos que a determinam, corroborando a concepção da determinação social do 
processo saúde-doença, na qual ele é entendido como resultado de fatores relacionados às formas de viver e trabalhar dos indivíduos, dos quais advêm potenciais de fortalecimento e desgaste da saúde (Moraes, 2006; Queiroz e Salum, 1996).

Essa nova abordagem do processo saúde-doença trouxe, portanto, a urgência de se reconhecer as necessidades de saúde do coletivo (a totalidade da população), a partir de sua inserção social em grupos homogêneos de certo território, em que está localizando o indivíduo e sua família (Queiroz e Salum, 1996).

Neste sentido, o território não é concebido apenas como área geográfica, mas sim, existencial, constituído pelos conflitos e interesses das pessoas que nele habitam, os quais por sua vez se relacionam com amigos, vizinhos, família, instituições e cenários (igreja, cultos, escola, trabalho, bares etc.); dessa forma, essa noção de território busca a organização de uma rede de atenção às pessoas com sofrimento psíquico, suas famílias, seus amigos e interessados (Silveira, 2003; Brasil, 2004).

No território, a construção de uma rede de atenção aos portadores de sofrimento psíquico, que melhore sua qualidade de vida, incluindo o cuidado sem estigmas e discriminação, é um dos grandes desafios enfrentados pelo SUS, no sentido de articular os serviços e iniciativas que possam responder às diferentes demandas que a doença ou a limitação apresentam para usuários e equipes de cuidados (Pitta, 2001).

Partindo desses objetivos, é essencial que este trabalho em rede seja delineado a partir da intersetorialidade, envolvendo não apenas os profissionais de saúde, mas também os profissionais de outras áreas, formando assim uma "unidade do fazer" associada a vinculação, solidariedade e complementaridade na ação humana (Mendes, 1999).

Dessa forma, pode-se pensar na reabilitação psicossocial do portador de sofrimento psíquico, porque ela pressupõe a penetração do usuário nas redes sociais da comunidade, utilizando os recursos vindos dela para restituir o poder contratual do usuário e ampliar a sua autonomia, que, por sua vez, implicaria na possibilidade de os sujeitos reconstruírem os sentidos de sua vida. Essa ressignificação teria peso efetivo no seu modo de viver, incluindo a luta pela satisfação de suas necessidades da forma mais ampla possível (Kinoshita, 2001; Cecílio, 2001).
Podemos dizer que a autonomia, enquanto necessidade de saúde no campo da saúde mental, só será produzida se houver estratégias de ação que caminhem ao encontro da história de vida dos portadores de sofrimento psíquico, da reconstrução de sua identidade, da valorização de suas individualidades, do reconhecimento dos seus direitos humanos (Amarante e Guljor, 2005; Pasquale, 2000).

Os pressupostos da Promoção da Saúde, em sintonia com essas questões, coadunam-se com todos os processos que interferem nas condições de vida e saúde da população, sejam eles econômicos, sociais, culturais, políticos, e apóiam-se na justiça social para assegurar condições dignas de sobrevivência para a população (Bydlowski e col., 2004).

Assim, diante da discussão que iniciamos e da riqueza do material empírico obtido a partir de entrevistas semiestruturadas com os gerentes dos equipamentos de saúde estudados, neste trabalho faremos um recorte do estudo que anteriormente realizamos no Distrito de Saúde, concentrando-nos, neste momento, em um equipamento da rede de atenção comunitária em saúde mental. Nosso objetivo é analisar as estratégias de produção de cuidado em saúde mental do CECCO na perspectiva de seu alinhamento com as políticas de saúde mental e promoção da saúde, verificando as potencialidades da interação entre elas, no cotidiano das práticas.

\section{As Políticas de Promoção da Saúde e Saúde Mental: interfaces}

De acordo com a Carta de Ottawa, oriunda da $1^{a}$. Conferência Internacional sobre Promoção da Saúde, realizada em Ottawa, Canadá, em 1996, “as ações de promoção da saúde [...] objetivam assegurar oportunidades e recursos igualitários para capacitar todas as pessoas a realizar completamente seu potencial de saúde" (Brasil, 2001, p. 21).

O conteúdo dessas ações inclui, portanto, “ambientes favoráveis, acesso à informação, a experiências e habilidades na vida, bem como oportunidades que permitam fazer escolhas por uma vida mais saudável" (Brasil, 2001, p. 21) e, nesse aspecto, fica clara a possibilidade de diálogo entre a promoção da saúde e a saúde mental, quanto à necessidade da autonomia do portador de sofrimento psíquico, das oportunidades 
que o capacitem a fazer escolhas, participando como protagonista na produção do seu cuidado; enfim, da retomada do lugar social desse usuário, num franco movimento de luta contra a exclusão.

A Política Nacional de Promoção da Saúde (Brasil, 2006), operando na diretriz da intersetorialidade, define a promoção da saúde como:

[...] um mecanismo de fortalecimento e implantação de uma política transversal, integrada e intersetorial, que faça dialogar as diversas áreas do setor sanitário, os outros setores do Governo, o setor privado e não governamental e a sociedade, compondo redes de compromisso e corresponsabilidade quanto à qualidade de vida da população em que todos sejam partícipes na proteção e no cuidado com a vida (p. 12).

Para essa política, a promoção da saúde é uma possibilidade de enfocar os aspectos que determinam o processo saúde-doença no país, como, por exemplo: violência, desemprego, subemprego, falta de saneamento básico, dificuldade de acesso à educação, fome etc., e, segundo a inspiração das estratégias propostas pela Carta de Ottawa, potencializar formas mais amplas de intervir em saúde, tomando como objeto os problemas e necessidades de saúde e seus determinantes e condicionantes, incidindo sobre as condições de vida e favorecendo as escolhas da população, reconhecendo, que:

[...] os modos como sujeitos e coletividades elegem determinadas opções de viver como desejáveis, organizam suas escolhas e criam novas possibilidades para satisfazer suas necessidades, desejos e interesses pertencem à ordem coletiva, uma vez que seu processo de construção dá-se no contexto da própria vida (Brasil, 2006, p. 7).

Nessas considerações, o que se destaca como objeto de reflexão, na aproximação da política de promoção da saúde com a política de saúde mental, diz respeito à estratégia de potencializar formas mais amplas de intervir em saúde, haja vista a pluralidade das necessidades de saúde do portador de sofrimento psíquico e o que preconiza o Ministério da Saúde, em relação à saúde mental: organizar uma rede de atenção às pessoas que sofrem com transtornos mentais, convocando todos os recursos afetivos, sanitários, sociais, econômicos, culturais e de lazer, para a produção do cuidado integral ao usuário (Brasil, 2006, 2004).
No Brasil, os substratos políticos e teóricos para a política de saúde mental, nos últimos anos, foram oferecidos pela III Conferência Nacional de Saúde Mental (Brasil, 2002), que contou com a participação ampla dos usuários e de seus familiares. Esse evento adotou como slogan "Cuidar, sim. Excluir, não", elaborando "propostas e estratégias para efetivar e consolidar um modelo de atenção em saúde mental que seja humano, de qualidade e com participação e controle social", buscando "transformar as formas de lidar com a experiência da loucura e da invalidação, e de produzir novas possibilidades de vida e de relações sociais" (p. 21).

Portanto, a política nacional de saúde mental, no contexto das possibilidades e limites do SUS, reafirma os desafios, os conflitos e as tensões que marcam a Reforma Psiquiátrica Brasileira, processo histórico compreendido como a transformação de práticas, saberes, valores sociais e culturais, através da implementação de projetos comprometidos com a afirmação de direitos de cidadania, a luta contra as desigualdades sociais e com os mecanismos de exclusão social (Brasil, 2002).

Segundo o relatório da III Conferência Nacional de Saúde Mental (Brasil, 2002):

A reorientação do modelo assistencial deve estar pautada em uma concepção de saúde compreendida como processo e não como ausência de doença, na perspectiva de produção de qualidade de vida, enfatizando ações integrais e promocionais de saúde (p. 24).

Aqui, portanto, reafirmamos a ligação entre a política de promoção da saúde e a de saúde mental, na perspectiva da Política Nacional de Promoção da Saúde (Brasil, 2006), quando ela refere que:

$\mathrm{O}$ aparato biomédico não consegue modificar os condicionantes nem determinantes mais amplos do processo saúde-adoecimento, operando um modelo de atenção e cuidado marcado, na maior parte das vezes, pela centralidade dos sintomas.

Assim, em consonância com essas políticas, é necessário que os profissionais de saúde se apropriem da compreensão do social, construindo estratégias operativas que ajudem a transformar a vida dos usuários com transtornos mentais, criando uma emancipação progressiva da doença, do estigma e da instituição (Pasquale, 200o), através da conformação de uma rede de 
cuidados em saúde mental, no território, que permita: a intervenção sobre as condições de vida da população, valorizando o processo de construção da saúde no “contexto da própria vida", segundo o último relatório de gestão em saúde mental no SUS (Brasil, 2007); a implementação de modalidades assistenciais que desenvolvam práticas pautadas em relações que potencializem a subjetividade, a autoestima, a autonomia e a cidadania, de acordo com o relatório da III Conferência Nacional de Saúde Mental (Brasil, 2002).

Entendemos, portanto, que a rede de atenção de base comunitária em saúde mental depende, como condição para se constituir, das ações intersetoriais no território, baseando-nos na definição de intersetorialidade concebida pela Política Nacional de Promoção da Saúde (Brasil, 2006) como:

Uma articulação das possibilidades dos distintos setores de pensar a questão complexa da saúde, de co-responsabilizar-se pela garantia da saúde como direito humano e de cidadania e de mobilizar-se na formulação de intervenções que a propiciem (p. 10).

A perspectiva da intersetorialidade, portanto, traz consigo a potência da integralidade das ações em saúde numa dimensão "macro" (Cecílio, 2001), porque extrapola os muros das organizações, problematizando os desafios cotidianos do cuidado e produzindo interações entre os diversos setores da sociedade.

Essa dimensão foi denominada de "integralidade ampliada” porque diz respeito à articulação de cada serviço de saúde a uma rede mais complexa, composta de outros serviços de saúde e outras instituições não necessariamente do setor "saúde", já que a problemática do sofrimento psíquico não é simples e o seu enfrentamento não deve contar com uma resposta apenas médica e localizada em um modelo único de serviço (Cecílio, 2001; Silveira, 2003).

Outra aproximação entre a política de saúde mental e a de promoção da saúde pode ser encontrada nos objetivos desta última, referentes à intersetorialidade: 1) ampliar a autonomia de sujeitos e coletividades, no cuidado integral à saúde, minimizando e/ou extinguindo as desigualdades de toda e qualquer ordem; 2) promover, entre os trabalhadores de saúde, o entendimento da concepção ampliada de saúde (Brasil, 2006).

Permeando toda essa discussão sobre a interação entre as duas políticas está a questão da inclusão social do usuário, traduzida, pela política de saúde mental, como a "construção de um novo lugar social para o chamado louco", através de um modelo de atenção em saúde mental que elabore novas referências conceituais para a questão da "loucura", do sofrimento mental e seu tratamento, sustentando a ideia do protagonismo e da cidadania de usuários e familiares (Brasil, 2007).

Na vertente da promoção da saúde, as ações sociais inclusivas se traduziriam pelo estímulo às alternativas inovadoras, que valorizem e otimizem o uso dos espaços públicos de produção de saúde e convivência para o desenvolvimento das ações de promoção da saúde (Brasil, 2006).

\section{Trajetória Metodológica}

\section{Local de Estudo}

O estudo foi realizado no CECCO que compõe a rede de atenção à saúde mental, de base comunitária, de um Distrito de Saúde (DS) localizado na zona norte do município de São Paulo, caracterizado por forte exclusão social da população, conforme constatamos na pesquisa anterior.

Segundo documento produzido pela Secretária Municipal de Saúde de São Paulo (São Paulo, 2004), esse DS possui 101 favelas, de um total de 2.018 no município de São Paulo, sendo que a taxa anual de crescimento da população favelada é de 3,68\%, superando a média de 2,97\% do município. Outros dados que corroboram o diagnóstico dessa exclusão dizem respeito à educação: $41,80 \%$ dos chefes de família possuem apenas o ensino fundamental completo, e a média de anos de estudo dos chefes de família é de 6,55; em comparação à totalidade do município, observamos que, nesse, 49,69\% dos chefes de família tem o ensino fundamental completo e sua média de anos de estudo é de 7,67 anos.

Dentre os chefes de família, $55,72 \%$ possuem rendimento de até 5 salários mínimos, enquanto,no total, o município de São Paulo possui 47,55\% de chefes de família com rendimento mensal de até 5 salários mínimos (São Paulo, 2004).

As ações de saúde do Programa Saúde da Família, com 42 equipes, apresentam uma taxa de cobertura de apenas 35,97\%. Esse Distrito de Saúde possui um dos piores índices de saúde do município, encontrando-se na $25^{\text {a }}$ posição do ranking entre as 31 Subprefeituras (São Paulo, 2004, 2005). 
Diante desses dados, portanto, podemos observar um quadro composto de uma rede de determinações sociais que expõe a população do território aos "potenciais de desgaste da saúde", concorrendo para a produção social do sofrimento psíquico (Queiroz e Salum, 1996; Lopes, 1999).

\section{Os Centros de Convivência e Cooperativa (CECCO)}

Os CECCOs foram implantados no município de São Paulo a partir de 1989, dentro da proposta de formação de uma rede que substituísse os manicômios, para servir como elo com comunidade, por meio de atividades para portadores de transtornos mentais, deficiências, idosos, crianças e adolescentes em situação de risco social, além de frequentadores habituais dos locais públicos e de livre circulação. Aliás, nesses locais é que essas ações preferencialmente se desenvolvem. Por tudo isso, o CECCO é considerado um serviço emblemático de promoção da inclusão, como opção das políticas públicas (Castanho, 2005).

O CECCO é conhecido por subverter os padrões conhecidos e clássicos de se executar saúde, introduzindo as artes, $o$ artesanato, o esporte, como linguagens que redesenham possibilidades de reabilitação psicossocial, já que a arte, a convivência e o trabalho cooperado aglutinam, em grupos heterogêneos, interesses, e não diagnósticos, buscando reinventar contratos nas relações humanas e institucionais, não mais sob a égide da doença e da tutela (Lopes, 1999).

Assim, como dispositivo de inclusão social do portador de sofrimento psíquico, o CECCO desenvolve iniciativas e práticas de saúde implicadas na redução das situações de desigualdades, estimulando ações intersetoriais e fortalecendo o empoderamento individual e comunitário (Brasil, 2006), diretrizes da Política Nacional de Promoção da Saúde, em interação com a política de saúde mental.

O CECCO em que realizamos este estudo foi fundado em 1991 e localizava-se em um centro esportivo. Mas a inadequação do espaço físico ocasionou sua transferência para o interior de uma Unidade Básica de Saúde (UBS). Ele funciona das sete da manhã às dezessete horas, e possui no quadro de funcionários, além de seu coordenador, um terapeuta ocupacional, um fonoaudiólogo, uma assistente social e uma fisioterapeuta.

\section{Tipo de pesquisa, coleta e análise de dados}

Após a aprovação do projeto pelo Comitê de Ética da
Escola de Enfermagem da Universidade de São Paulo, iniciamos este estudo, de abordagem qualitativa. Os procedimentos qualitativos são utilizados para que o investigador verifique como as pessoas avaliam uma experiência, ideia ou evento; como definem um problema e quais as opiniões, os sentimentos e significados que se encontram associados a determinados fenômenos (Iervolino e Pelicioni, 2001).

A coleta de dados foi realizada a partir de entrevista semiestruturada com o coordenador do CECCO, opção que se baseou em nosso entendimento sobre a relevância do papel da gerência em saúde. Em consonância com alguns autores, acreditamos que o papel da gerência em saúde seja organizar a produção das ações de saúde dos serviços, buscando o conjunto das necessidades de saúde da coletividade, através de uma importante atividade integrativa e articuladora, cujo caráter de negociação implica lidar com as relações de trabalho na equipe de saúde e nas relações da unidade com a comunidade (Mishima e col., 1997). Sendo assim, a gerência em saúde é um instrumento necessário à consolidação do SUS, que por meio de suas políticas públicas, traduz a complexidade e a abrangência do campo da saúde mental.

Portanto, optamos por ouvir o coordenador do CECCO para que o estudo não se fundamentasse apenas na literatura e nos documentos oficiais do Ministério da Saúde, mas que também pudesse promover o diálogo entre estes e os depoimentos obtidos pela gerência do serviço; logo, os últimos seriam utilizados como contraponto e/ou complemento dos achados teóricos.

Dessa forma, o instrumento de coleta de dados que aplicamos era composto de questões norteadoras, que versavam sobre as estratégias de atendimento do serviço no campo das necessidades de saúde mental e a articulação do serviço com a rede de atenção em saúde mental do território. A entrevista foi gravada e posteriormente transcrita para a construção de um texto que se integrava com os documentos oficiais do Ministério da Saúde acerca das políticas de saúde mental e promoção da saúde no Brasil, considerando a importância desse órgão na formulação e no financiamento das políticas de saúde do país, no sentido da transformação do modelo de atenção em saúde mental, logrando atingir os princípios doutrinários e operacionais do SUS na perspectiva da consolidação da Reforma Psiquiátrica Brasileira. 


\section{Resultados e Discussão}

No que diz respeito à concepção do processo saúde-doença, observamos, através da fala do coordenador, que este serviço relaciona a grande demanda de saúde mental do território ao desemprego e à violência, marcantes no Distrito de Saúde, no qual os homicídios ocupam a terceira posição entre as cinco maiores causas de óbito (São Paulo, 2004), o que espelha a marginalização social em que vive sua população:

Hoje a gente vê que [...] as pessoas adoecem porque perderam o emprego, porviolência. Aquiéum lugar de altíssimo índice de violência.

Portanto, vimos que, na concepção do trabalhador de saúde entrevistado, atrelada à determinação social do processo saúde doença, os problemas e as necessidades de saúde do usuário portador de sofrimento psíquico são decorrentes das formas como ele se insere na reprodução social (Campos e Soares, 2003) e, portanto, o sofrimento psíquico é entendido como uma produção social de determinação múltipla e complexa (Brasil, 2006).

Estreitando o modelo da determinação social do processo saúde-doença ao paradigma da promoção, vimos convergência na visão sobre os fatores que colocam a saúde da população em risco e na forma como as diferenças entre esses fatores são dimensionadas a depender do território do país, gerando para cada um deles distintas necessidades de saúde (Brasil, 2006).

Assim, a compreensão do sofrimento psíquico aproxima a política da promoção da saúde e de saúde mental, porque pressupõe ações para transformar as condições de produção do sofrimento, estratégia proposta pela III Conferência Nacional de Saúde Mental (Brasil, 2002).

Quanto às estratégias de atendimento em saúde mental do CECCO, de acordo com o sujeito de pesquisa entrevistado, o serviço mantém sua "porta aberta”, ou seja, mesmo que o usuário chegue sem o encaminhamento de uma unidade de saúde é acolhido e passa por uma entrevista com os profissionais de saúde, sendo já incluído em alguma atividade, dentre as oficinas terapêuticas.

Segundo relato, são raros os casos de demanda reprimida pelo CECCO; quando ocorre, é por causa da severidade das co-morbidades apresentadas pelo usuário, o que dificulta muito sua inserção nas atividades oferecidas, já que necessita de cuidados especiais.
Além disso, o CECCO desenvolve ações de promoção da saúde em parceria com o Centro de Atenção Psicossocial (CAPS) do território, nas quais esses dois serviços extrapolam seus muros e desenvolvem atividades conjuntas nos espaços de convivência, cultura, lazer e esporte do território, como a casa de cultura, o centro esportivo em que se desenvolvem jogos, a oficina de tapeçaria. Através dessa estratégia, o CECCO pretende captar o usuário do CAPS que está saindo da crise, para que ele já comece a participar de algumas oficinas, já que grande parte da demanda que esse serviço recebe é composta de pessoas que estiveram em tratamento no CAPS e obtêm melhora.

Por localizar-se no mesmo espaço físico de uma Unidade Básica de Saúde (UBS), o CECCO realiza, então, outra parceria no atendimento à população, articulando-se, portanto, com a Atenção Básica à Saúde. Assim, desenvolve, em conjunto com a UBS, o acolhimento ao portador de sofrimento psíquico:

Aqui a gente tem uma experiência interessante por estar num posto e ter uma população muito geral, que vem aqui procurar o médico porque está, sei lá, deprimido; aí acaba conhecendo as atividades do CECCO e muitas vezes não vai mais pro serviço médico, né, acabam dando uma resolução para o que vieram buscar, na verdade muitas vezes era só conversar com alguém, então acabam saindo daquela coisa mais doença...

Essas experiências, de acordo com o que foi relatado, além de se configurarem em ações interdisciplinares em saúde, envolvendo os profissionais do CECCO, do CAPS e da UBS, potencialmente permitem a superação do modelo biomédico - centrado na doença e na intervenção essencialmente farmacológica para a terapêutica dos transtornos mentais - o que sinaliza a direção desses três serviços (CECCO, CAPS e UBS) para práticas voltadas à promoção de saúde.

Com isso, podemos observar a importância da articulação entre a UBS e os serviços que compõem a rede de atenção em saúde mental, substituindo o hospital psiquiátrico; essa estratégia se alinha ao que é preconizado pela política de saúde mental do SUS, segundo a qual esses serviços substitutivos devem ser territorializados e integrados à rede de saúde que realize ações de proteção, promoção, prevenção e recuperação em saúde mental (Brasil, 2002). 
As ações do CECCO constituem-se em oficinas de atividades, que são bastante variadas, para que o sujeito possa escolher com as quais se identifica. Dessa forma, as oficinas dirigem-se às pessoas não pelo seu diagnóstico; ao contrário, estão à disposição do usuário segundo o seu desejo de participação, através das possibilidades de escolha que a ele são oferecidas.

Segundo o profissional entrevistado, as oficinas:

[...] são um meio de trabalhar o aprendizado e o desenvolvimento do potencial criativo, sendo uma estratégia mesmo de sociabilidade, de convivência, de resgate da cidadania, de ser alguém, de ter um lugar, então eu acho que elas são bastante resolutivas, a gente vê, assim, na prática.

Portanto, a produção do cuidado ao portador de sofrimento psíquico ocorre a partir do reconhecimento da subjetividade do usuário, explorando suas potencialidades criativas; do desenvolvimento de atividades educativas que democratizem o conhecimento, visando ao empoderamento do portador de sofrimento psíquico, para capacitá-lo a efetuar suas próprias escolhas; da convivência como forma de esses sujeitos resgatarem sua inserção numa rede social que, em decorrência do sofrimento psíquico severo, está fragilizada (Buss, 2000; Fortes e Sacardo, 1999).

Os CECCO foram criados para dar, na verdade, inclusão, resgate da cidadania, da convivência, porque essas pessoas, com o tempo, com a cronificação, por serem internadas várias vezes, perdiam essa rede social, então ficavam com uma rede social bastante estilhaçada; por isso os CECCOs foram criados em espaço público, por excelência, parques, centros esportivos... (coordenador do CECCO).

Percebemos, então, que o CECCO visa ampliar a autonomia do portador de sofrimento psíquico, indo contra uma visão assistencialista, que reforça a dependência, e interagindo com a política nacional de promoção da saúde, porque suas ações visam a minimizar e/ou extinguir as desigualdades, além de valorizar e otimizar o uso dos espaços públicos de convivência e de produção de saúde para o desenvolvimento das ações de promoção da saúde (Brasil, 2006).

Segundo a III Conferência Nacional de Saúde Mental (Brasil, 2002), na direção dos processos de produção de autonomia e de direitos de cidadania do usuário com sofrimento psíquico, "o processo de Reforma Psiquiá- trica requer a implementação de políticas públicas que garantam e consolidem a criação de Programas de Geração de Renda e Trabalho e de Cooperativas e Associações de Usuários" (p. 45). Com esse objetivo, foram aprovadas as propostas de considerar ações que visam o trabalho com geração de renda como parte integrante do cuidado em saúde mental, que devem ser incentivadas para o enfrentamento do desemprego, da miséria e da exclusão social.

As oficinas de trabalho cooperado do CECCO visam à inserção do usuário portador de necessidades especiais no mercado de trabalho, com uma produção qualificada a ser comercializada - e não como uma atitude assistencialista de "inclusão consentida" no trabalho e, portanto, sua preocupação é revelar potencialidades, na produção de sentido no trabalho desempenhado e no compromisso com a produção, considerando o limite diferenciado de cada usuário na avaliação qualitativa e quantitativa para uma divisão igualitária dos benefícios (Lopes, 1999).

Nessa perspectiva, o CECCO estudado desenvolve uma parceria com uma associação - que para preservar a identidade do sujeito de pesquisa será denominada com o codinome "Associação Y" - a qual possui um núcleo de geração de renda e trabalho. Essa organização reúne voluntários, familiares, usuários e trabalhadores de equipamentos de saúde mental da região norte do município de São Paulo:

Com a Associação Y montou-se um grupo que é muito interessante, que dá mesmo condição dessa pessoa sair e arrumar um emprego, agora a gente está tentando uma parceria com o Fundo de Solidariedade lá do Estado, porque eles dão o certificado, então se a gente consegue ser cadastrado os usuários recebem um certificado...

Assim, de acordo com as diretrizes da Política Nacional de Promoção da Saúde (Brasil, 20o6), vimos que o CECCO com ela interage, então, por "estimular as ações intersetoriais, buscando parcerias que propiciem o desenvolvimento integral das ações de Promoção da Saúde", além de "fortalecer a participação social como fundamental na consecução de resultados de promoção da saúde, em especial a equidade e o empoderamento individual e comunitário" (p. 15).

A participação da comunidade nas ações de saúde ocorre também por meio das oficinas terapêuticas, de trabalhadores voluntários, que, juntamente com um 
profissional de saúde mental, participam das oficinas e ensinam aos usuários alguma atividade:

Nós temos muitos voluntários da comunidade, $e$ isso é bem legal, então é a pessoa que sabe fazer... um artesanato, por exemplo, e quer ensinar. Aífica um técnico de saúde junto porque, como os usuários são mesclados, pode acontecer de uma pessoa não estar muito bem naquele dia, né, muitas vezes começar a ter problemas, a ter um surto. $O$ técnico trabalha na verdade a dinâmica de grupo, ele não ensina a técnica, a gente promove a inserção, né? (coordenador do CECCO)

Portanto, observamos que a prática intersetorial, na qual se engaja o CECCO, contribui para o trabalho de equipe e a amplitude de ação de muitos atores na resposta ao sofrimento mental e à exclusão (Lopes, 1999).

\section{Conclusão}

Através do diálogo com as políticas de saúde mental e de promoção da saúde, "recortadas" a partir dos documentos oficiais do Ministério da Saúde, e a proposta do CECCO estudado, concluímos que este serviço - por meio de suas ações e estratégias que foram foco deste artigo, direcionadas ao portador de sofrimento psíquico - opera na autonomia e na cidadania dessas pessoas, com base na diretriz da intersetorialidade.

A produção da autonomia e cidadania está presente nos depoimentos, tendo em vista o contexto de exclusão social do Distrito de Saúde e o reconhecimento da pluralidade dos determinantes do processo saúde-doença mental, e a intersetorialidade se apresenta como componente fundamental na construção/operacionalização de uma rede de atenção de base comunitária em saúde mental.

Além disso, através da experiência do CECCO, que acumula experiências positivas rumo à reabilitação psicossocial do usuário em sofrimento psíquico, pudemos verificar que as políticas de saúde mental e de promoção da saúde, interagindo de forma articulada, produzem potenciais de fortalecimento à saúde integral do usuário, tornando o CECCO um lugar de produção de sentido para a vida das pessoas por ele acolhidas.

\section{Referências}

AMARANTE, P. D. C.; GULJOR, A. P. Reforma psiquiátrica e desinstitucionalização: a (re) construção da demanda no corpo social. IN: MATTOS, R. A.; PINHEIRO, R. (Orgs.). Construção social da demanda. Rio de Janeiro: IMS,UERJ/ Cepesc/Abrasco, 2005. p. 65-76.

BRASIL. Ministério da Saúde. Política nacional de promoção da saúde. Brasília, 2006.

BRASIL. Ministério da Saúde. Promoção da saúde: Declaração de Alma-Ata, Carta de Ottawa, Declaração de Adelaide, Declaração de Sundsvall, Declaração de Santafé de Bogotá, Declaração de Jacarta, Rede de Megapaíses e Declaração do México. Brasília, 2001.

BRASIL. Ministério da Saúde. Relatório final da III Conferência Nacional de Saúde Mental. Brasília, 2002.

BRASIL. Ministério da Saúde. Saúde mental no SUS: acesso ao tratamento e mudança do modelo de atenção: Relatório de Gestão 2003-2006. Brasília, 2007.

BRASIL. Ministério da Saúde. Saúde mental no SUS: os centros de atenção psicossocial. Brasília, 2004.

BUSS, P. M. Promoção da saúde e qualidade de vida. Ciência \& Saúde Coletiva, Rio de Janeiro, v. 5, n.1, p. 163-77, 2000.

BYDLOWSKI, C. R.; WESTPHAL, M. F., PEREIRA, I. M. T. B. Promoção da saúde: porque sim e porque ainda não! Saúde e Sociedade, São Paulo, v. 13, n. 1, p. 14-24, jan./abr. 2004.

CAÇAPAVA, J. R.; COLVERO, L. A. Estratégias de atendimento em saúde mental nas unidades básicas de saúde. Revista Gaúcha de Enfermagem, Porto Alegre, v. 29, n. 4, p. 573-8o, dez. 2008.

CAMPOS S. M. S.; SOARES, C. B. A produção de serviços de saúde mental: a concepção de trabalhadores. Ciência \& Saúde Coletiva, Rio de Janeiro, v. 8, n. 2, p. 621-8, 2003. 
CASTANHO, P. C. G. O laço do preconceito: a inclusão, exclusão e convivência do usuário de saúde mental a partir do conceito de alianças inconscientes e do dia a dia de um CECCO. Vínculo, São Paulo, v. 2, n. 2, p. 70-9, dez. 2005.

CECÍlIO, L. C. O. As necessidades de saúde como conceito estruturante na luta pela integralidade e equidade na atenção em saúde. In: MATTOS, R. A.; PINHEIRO, R. (Orgs.). Os sentidos da integralidade na atenção e no cuidado à saúde. Rio de Janeiro: IMS,UERJ/Cepesc/Abrasco, 2001. p. 113-50.

FORTES, P. A. C. F.; SACARDO, D. P. Ética na assistência à saúde do adolescente e do jovem. In: BRASIL. Ministério da Saúde. (Org.). Cadernos juventude, saúde e desenvolvimento. Brasília, 1999. p. 147-61.

IERVOLINO, A. S.; PELICIONI, M. C. F. A utilização do grupo focal como metodologia qualitativa na promoção de saúde. Revista da Escola de Enfermagem da USP, São Paulo, v. 35, n. 2, p. 115-21, jun. 2001.

KINOSHITA, R. T. Contratualidade e reabilitação psicossocial. In: PITTA, A. (Org.). Reabilitação psicossocial no Brasil. São Paulo: Hucitec, 2001. p. 55-9.

LOPES, I. C. A contribuição paulistana à reforma em saúde mental brasileira. In: VIEIRA, M. C. T.; VICENTIN, M. C.; FERNANDES, I. A. (Orgs.). Tecendo a rede: trajetórias da saúde mental em São Paulo 1986-1996. Taubaté: Cabral Editora Universitária,1999. p. 28-78.

MENDES, E. V. Uma agenda para a saúde. São Paulo: Hucitec, 1999.
MISHIMA, S. M. et al. Organização do processo gerencial no trabalho em saúde pública. In: ALMEIDA, M. C. P.; ROCHA, S. M. M. (Orgs.). $O$ trabalho de enfermagem. São Paulo: Cortez, 1997. p. 261-96.

MORAES, D. R. Revisitando as concepções de integralidade. Revista de APS, Juiz de Fora, v. 9, n. 1, 2006. Disponível em: <http://www.nates.ufjf.br/novo/ revista/voognı.htm>. Acesso em: 1 fev .2008.

PASQUALE, E. Gestão da psiquiatria na comunidade. Unopar Científica: Ciências Biológicas e da Saúde, Londrina, v. 2, n.1, p. 27-34, 2000.

PITTA, A. Reorientação do modelo de atenção: equidade e justiça social na organização de serviços de saúde mental. In: BRASIL. Ministério da Saúde. Conselho Nacional de Saúde. Caderno de textos da III Conferência Nacional de Saúde Mental: cuidar sim, excluir não. Brasília, 2001. p. 20-30.

QUEIROZ, V. M.; SALUM, M. J. L. Reconstruindo a intervenção de enfermagem em saúde coletiva face à vigilância à saúde. In: CONGRESSO BRASILEIRO DE ENFERMAGEM, 48., 1996, São Paulo. Anais... São Paulo: ABEn-Seção SP, 1996. p. 347.

SÃO PAULO (Cidade). Secretária Municipal da Saúde. Índice Saúde 2005. São Paulo, 2005.

SÃO PAULO (Cidade). Secretária Municipal da Saúde. Sumário de Dados 2004. São Paulo, 2004. SILVEIRA, D. P. Sofrimento psíquico e serviços de saúde: uma cartografia da produção do cuidado de saúde mental na atenção básica de saúde. 2003.184 p. Dissertação (Mestrado em Saúde Pública) - Escola Nacional de Saúde Pública, Rio de Janeiro, 2003. 